\title{
Tuning the thermal conductance of molecular junctions with interference effects
}

\author{
J. C. Klöckner ${ }^{1}{ }^{*}$ J. C. Cuevas ${ }^{1,2}$, and F. Pauly ${ }^{1,3}$ \\ ${ }^{1}$ Department of Physics, University of Konstanz, D-78457 Konstanz, Germany \\ ${ }^{2}$ Departamento de Física Teórica de la Materia Condensada and Condensed Matter Physics Center (IFIMAC), \\ Universidad Autónoma de Madrid, E-28049 Madrid, Spain and \\ ${ }^{3}$ Okinawa Institute of Science and Technology Graduate University, Onna-son, Okinawa 904-0395, Japan
}

\begin{abstract}
We present an $a b$ initio study of the role of interference effects in the thermal conductance of single-molecule junctions. To be precise, using a first-principles transport method based on density functional theory, we analyze the coherent phonon transport in single-molecule junctions made of several benzene and oligo-phenylene-ethynylene derivatives. We show that the thermal conductance of these junctions can be tuned via the inclusion of substituents, which induces destructive interference effects and results in a decrease of the thermal conductance with respect to the unmodified molecules. In particular, we demonstrate that these interference effects manifest as antiresonances in the phonon transmission, whose energy positions can be tuned by varying the mass of the substituents. Our work provides clear strategies for the heat management in molecular junctions and more generally in nanostructured metal-organic hybrid systems, which are important to determine, how these systems can function as efficient energy-conversion devices such as thermoelectric generators and refrigerators.
\end{abstract}

\section{INTRODUCTION}

The manipulation of phonon heat conduction at the nanoscale is of fundamental interest for technologies such as thermoelectrics and thermal management in nanoelectronics ${ }^{1}$. Earlier attempts to perform such a manipulation focused on incoherent transport mechanisms, but in recent years, and with the advent of nanoscale devices and nanostructured materials, a great effort is being devoted to control heat currents in the coherent regime by making use of interference effects ${ }^{2}$. Thus for instance, in the context of phononic structures the so-called superlattices have been introduced. Here one can tune the phonon band structure, phonon group velocity, and the related phonon density of states by means of wave interference effects ${ }^{3,4}$. This strategy is limited by the quality of interfaces in terms of interface roughness and by the mean free path of the heat carriers. Due to these constraints the strategy is in general better applicable to systems, in which heat is carried by low-energy or equivalently long-wavelength phonons. However, if one assumes that atomically thin, single crystal planes can be manipulated at will, also higher-energy or short-wavelength phonons can in principle be affected, since structural length scales are of interatomic distance. Thus, Han et $a l . .^{5}$ theoretically proposed the suppression of heat transport using defect atom arrays embedded in a single crystal plane. To inhibit the heat flow, the authors exploited the destructive interference between two phonon paths. Resulting line shapes of the energy-dependent phonon transmission are indeed reminiscent of Fano resonances. Such Fano resonances are, in fact, a very general concept in nanostructured materials and occur in every system, in which a narrow discrete mode couples to a broad continuous spectrum ${ }^{6}$. Indeed, they have been reported in a great variety of systems ranging from quantum dots to photonic structures ${ }^{7-9}$.

As compared to the inorganic materials discussed above, molecules offer an ideal platform to tailor structures at the single-atom level, and single-molecule junctions can be used to probe the coherent transport through the molecules ${ }^{10}$. In the field of molecular electronics interference effects have been studied with a special emphasis on their impact on the electronic transport properties of such junctions. Theoretically this topic has been explored extensively in the last two decades ${ }^{11-27}$. In particular, special attention has been devoted to the role of Fano resonances ${ }^{28-32}$ and to the determination of general rules governing the appearance of quantum interference effects in molecules with extended $\pi$-electron systems $^{33-36}$. Also different experimental reports in recent years have convincingly shown the influence of the quantum interference on electronic transport of molecular junctions, as illustrated by measurements of linear conductances and current-voltage characteristics ${ }^{37-48}$. When comparing electron and phonon transport, it needs to be kept in mind that for electrons the interference has to occur within some $k_{\mathrm{B}} T$ around $\mu \approx E_{\mathrm{F}}$, with the Fermi energy $E_{\mathrm{F}}$, to get a measurable effect on the linear conductance. For phonons instead, Debye energies of typical metal electrodes are in the range of several ten meV so that already a sizable window of phonon energies contributes to thermal transport at room temperature.

In the case of coherent phonon transport in molecular junctions the impact of interference effects is starting to be analyzed theoretically ${ }^{49,50}$. For instance, Markussen $^{49}$ investigated the role of phonon interference in molecular junctions made of benzene and oligo-phenylene-ethynylene (OPE3) molecules attached to $\mathrm{Si}$ and graphene nanoribbon electrodes. Combining ab initio calculations for vibrational properties of the molecules with a phenomenological description of the leads and the molecule-lead couplings, Markussen found that the phonon transmission function for crossconjugated molecules, like meta-connected benzene, exhibits destructive interference features very similar to 
those found for the corresponding electronic transport, which cause a reduction of the phononic thermal conductance with respect to the linearly conjugated analogues. On the other hand, Famili et al. ${ }^{50}$ studied the phonon transport in alkane chains by means of a first-principles method based on density functional theory (DFT). In particular, they investigated the appearance of Fano resonances, when the alkanes are modified by the inclusion of certain side groups, so-called "Christmas trees". These resonances led to a reduction of the corresponding thermal conductance by a factor 2 . However, alkanes are known to exhibit geometrical gauche defects that result in the localization of vibrational modes. These defects have been reported to reduce the thermal conductance by a similar magnitude ${ }^{51}$, making it presumably difficult to discriminate between the effects of side-groups and gauche defects. In this sense, the study of stiff molecules like benzene derivatives may provide more conclusive results about the existence of interference effects.

In this work we employ a full ab initio DFT-based transport method to study the role of phonon interference effects in the thermal conductance of single-molecule junctions. In particular we explore benzene- and OPE3related molecules, but contrary to Ref. [49] we assume that the electrodes are made of $\mathrm{Au}$, which is often the material of choice for the leads of molecular junctions, and use amine anchoring groups. In the case of benzenediamine we find that due to the small Debye energy of $\mathrm{Au}$ of around $20 \mathrm{meV}$, no destructive interference effects are visible in the phonon transmission function, irrespective of whether the molecule is contacted to $\mathrm{Au}$ in para or meta configuration. This leads to a room-temperature thermal conductance that is similar for the two contacting schemes. More importantly we show that this situation can be changed by replacing a $\mathrm{H}$ atom of benzene by a halogen atom (F, Cl, Br, I). The substitution may lead to a reduction of the thermal conductance by up to a factor of 1.7. We also show that by increasing the number of substituent atoms in the benzene molecule and depending on their precise position on the ring, one can induce additional reductions of the thermal conductance by a factor of 2.5. Finally, we also show that similar concepts apply to the case of OPE3 and, in particular, we find a clear difference between para- and meta-OPE3, where the central benzene ring is connected in para or meta position. Our work provides concrete predictions that can potentially be tested, given the recent experimental advances in the measurement of the thermal conductance of atomic-scale contacts ${ }^{52,53}$. In addition, it sheds light on the importance of phonon interference effects to tune the thermal transport of molecular junctions.

The rest of the paper is organized as follows. In section II we briefly describe the theoretical techniques employed in this work to study the phonon transport in singlemolecule junctions. In section III we present the main results of this work concerning the phonon thermal conductance of single-molecule junctions based on benzene and OPE3 derivatives. We summarize our main conclu- sions in section IV. Finally, we discuss in Appendix A our results for the electronic thermal conductance of the molecular junctions studied in this work to show that the thermal transport is actually dominated by phonons.

\section{THEORETICAL METHOD}

To explore the influence of phonon interference on the thermal conductance of single-molecule junctions, we describe coherent phonon transport within the LandauerBüttiker approach. This approach is based on the harmonic approximation and is valid, if the characteristic dimensions of the atomic-scale junction are smaller than the inelastic mean free path for phonons, which is on the order of a few $\mathrm{nm}$ at room temperature for gold ${ }^{54}$. The main source of resistance is then the elastic scattering in the narrowest part of the device. Thermalization due to third or higher order interactions between atoms that lead to phonon-phonon scattering is assumed to take place exclusively in the electrodes, which are thus treated as reservoirs with a well-defined thermodynamic state.

Within this approach the linear thermal conductance due to phonons is given by

$$
\kappa_{\mathrm{pn}}(T)=\frac{1}{h} \int_{0}^{\infty} d E E \tau_{\mathrm{pn}}(E) \frac{\partial n(E, T)}{\partial T},
$$

where $n(E, T)=\left[\exp \left(E / k_{\mathrm{B}} T\right)-1\right]^{-1}$ is the Bose function and $\tau_{\mathrm{pn}}(E)$ is the energy-dependent phononic transmission. We compute the transmission function $\tau_{\mathrm{pn}}(E)$ by means of a combination of DFT and non-equilibrium Green's function techniques, as we have described in detail in Refs. [55-57].

Briefly, the first step in the calculation is the construction of the molecular junction geometries. For this purpose, we use DFT to obtain equilibrium geometries through total energy minimization. From these calculations the vibrational properties in terms of the dynamical matrix are obtained by applying density functional perturbation theory, as implemented in the quantum chemistry software package TURBOMOLE $6.5^{58-60}$. In our DFT calculations we employ the Perdew-BurkeErnzerhof exchange-correlation functional ${ }^{61,62}$, the basis set of split-valence-plus-polarization quality def2$\mathrm{SV}(\mathrm{P})^{63}$, and the corresponding Coulomb fitting basis ${ }^{64}$. In order to accurately determine the force constants and related vibrational energies, we use very strict convergence criteria. In particular, total energies are converged to a precision of better than $10^{-9}$ a.u., whereas geometry optimizations are performed until the change of the maximum norm of the Cartesian gradient is below $10^{-5}$ a.u. We have checked that our stringent convergence criteria avoid the appearance of any modes with imaginary frequencies in the optimized junction region, which would otherwise signal unstable geometries. It is furthermore worth stressing that in our method the electrodes are described by means of perfect semi-infinite crystals, whose phonon properties are determined within DFT with the 
same functional and the same basis set as used for the central device part. In this way we achieve a consistent, full $a b$ initio treatment of the phonon system of the whole molecular junctions. Finally, the dynamical matrix of the molecular junction is used to compute the phonon transmission function with the help of non-equilibrium Green's function techniques, as presented in Ref. [55].

\section{RESULTS}

We start our discussion of the results with an analysis of single-molecule junctions based on the unsubstituted benzenediamine molecule. As shown in the upper part of Fig. 1, we consider contacts, where the amino $\left(\mathrm{NH}_{2}\right)$ group is attached to a single tip atom of the gold electrodes on each side both in the para and in the meta configuration. We will refer to this amino binding site on the gold also as "atop position" and note that these geometries are similar to those used in previous studies of electronic transport, mimicking typical binding geometries ${ }^{65}$. In Fig. 1(a) we show the results for the phononic transmission of these two binding configurations, computed with our ab initio method, described in the previous section. The first thing to notice is that the transmission is only finite below approximately $20 \mathrm{meV}$, which corresponds to the Debye energy of the gold electrode material. On the other hand, notice that although both transmission curves are different, which is reasonable due to the different geometrical configurations, we do not find any signature of destructive interference in the form of antiresonances. This is further confirmed by the results for the temperature dependence of the phononic thermal conductance, which we show in Fig. 1(b). In fact, both molecules exhibit similar thermal conductance values over the whole temperature range explored here.

The reason for the lack of destructive interference effects in these benzene-based junctions can be understood with the help of the work of Markussen ${ }^{49}$. Considering his semi-empirical results for phonon transport in benzene junctions with Si electrodes, he found that the lowest observable destructive interference features appear at energies around $40 \mathrm{meV}$. Since this energy is above the Debye energy of gold, no effects are visible in our case.

These results raise the question, whether it is possible to observe interference effects in the phonon transport in junctions based on benzene derivatives with the standard Au leads. In the context of electronic transport it is known that interference features can be shifted in energy by introducing side groups ${ }^{22}$, which have either electron-withdrawing or electron-donating character. The inclusion of such side groups moves the resonance features to lower or higher energies, respectively. Furthermore, the substituents can also break the symmetry of the molecule, leading to destructive interference in benzene junctions even for the para configuration of anchoring groups. Inspired by this idea, we shall analyze in what follows the effect of substituting a $\mathrm{H}$ atom in the
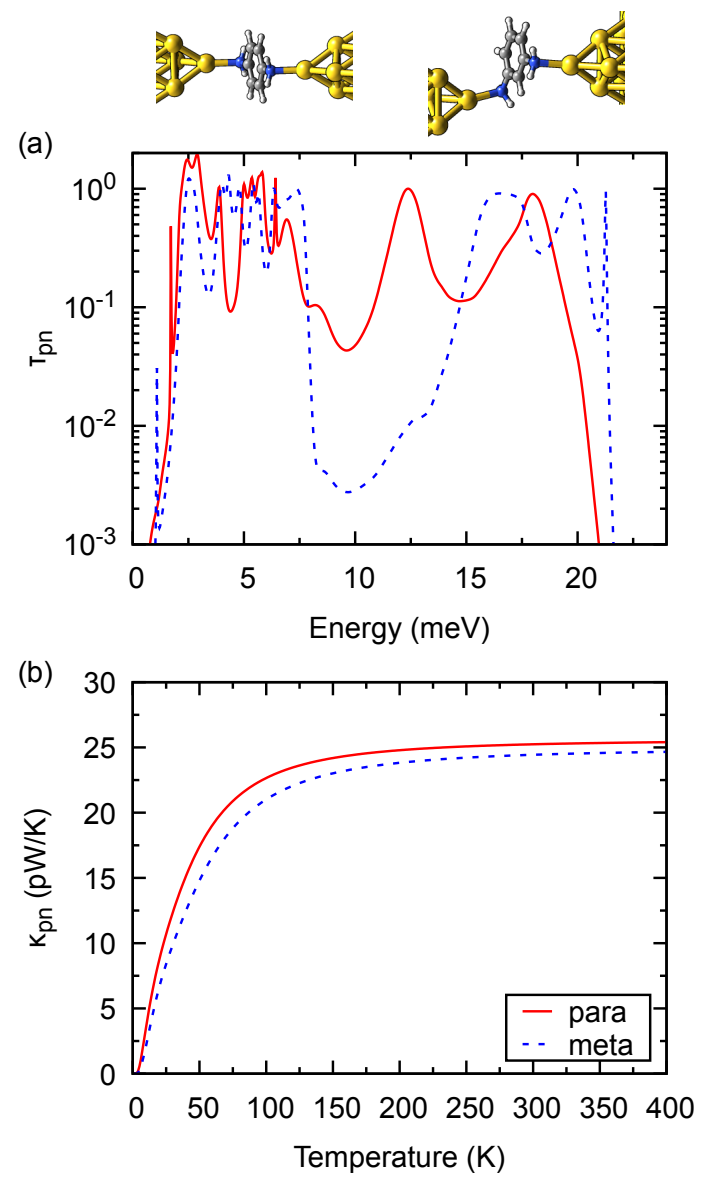

FIG. 1. (a) Phonon transmission as a function of energy for the Au-benzenediamine-Au junctions shown above this panel for both para (left) and meta (right) binding configurations. (b) The corresponding phononic thermal conductance as a function of temperature. The legend in panel (b) applies also to panel (a).

benzenediamine molecule by a heavier atom of mass $m$ to tune the position of the resonance features. The basic idea is to try to shift the destructive resonances below the Debye energy of gold to observe a measurable effect on the thermal conductance.

With this idea in mind we consider the phonon transport in Au-benzenediamine-Au single-molecule junctions, where one of the $\mathrm{H}$ atoms of the benzene has been substituted by a halogen atom $X=\mathrm{F}, \mathrm{Cl}, \mathrm{Br}, \mathrm{I}$, as depicted in the upper part of Fig. 2. The naive expectation is that since the energy of a harmonic oscillator scales as $E \propto \sqrt{k / m}$, with $k$ being the force constant, the resonance features should decrease in energy with increasing mass $m$ of the substituent from $\mathrm{F}$ to $\mathrm{I}$. This simple view is indeed confirmed by our ab initio calculations of the phononic transmission, which are summarized in Fig. 2(a) for the substituted benzenediamine molecule in the para configuration. As one can see, there is a clear destructive interference feature for $X=\mathrm{Br}$ at an energy of around $19 \mathrm{meV}$. It is further shifted to lower ener- 


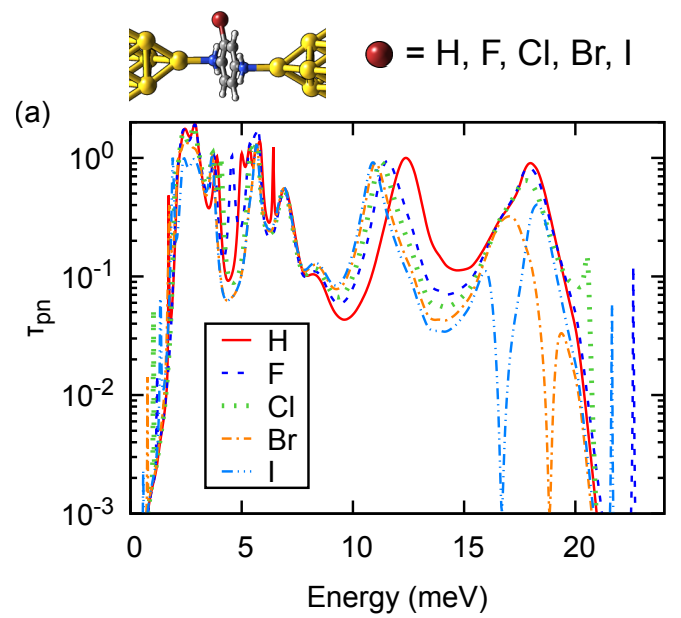

(b)

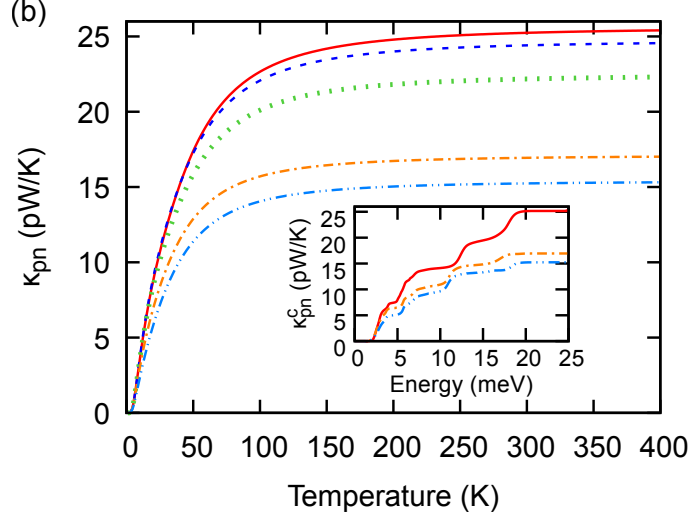

FIG. 2. (a) Phonon transmission as a function of energy for Au-benzenediamine-Au junctions, where a $\mathrm{H}$ atom of the benzene molecule has been substituted by a halogen atom $(X=\mathrm{F}$, $\mathrm{Cl}, \mathrm{Br}, \mathrm{I}$ ), see upper part of the figure. (b) The corresponding phononic thermal conductance as a function of temperature for the different benzenediamine derivatives. The inset shows the room-temperature cumulative thermal conductance as a function of energy for the junctions with $X=\mathrm{H}, \mathrm{Br}$, I. The legend in panel (a) applies also to panel (b) and its inset.

gies for $X=\mathrm{I}$, where it appears at around $16 \mathrm{meV}$. Additionally, we see that the peak at around $13 \mathrm{meV}$ for the unsubstituted benzenediamine shifts to lower energies as the mass of the substituent increases, while the transmission for energies lower than $10 \mathrm{meV}$ remains nearly unaffected. These results for the transmission have a clear impact on the phononic thermal conductance, see Fig. 2(b). Notice, in particular, that the thermal conductance decreases monotonically with the mass of the substituent, reaching in the case of $X=\mathrm{I}$ a reduction factor of 1.7 at room temperature, as compared with the unsubstituted benzene molecule.

To assess in a quantitative manner the impact of the antiresonances on the reduction of the thermal conductance upon the introduction of substituents, it is useful to

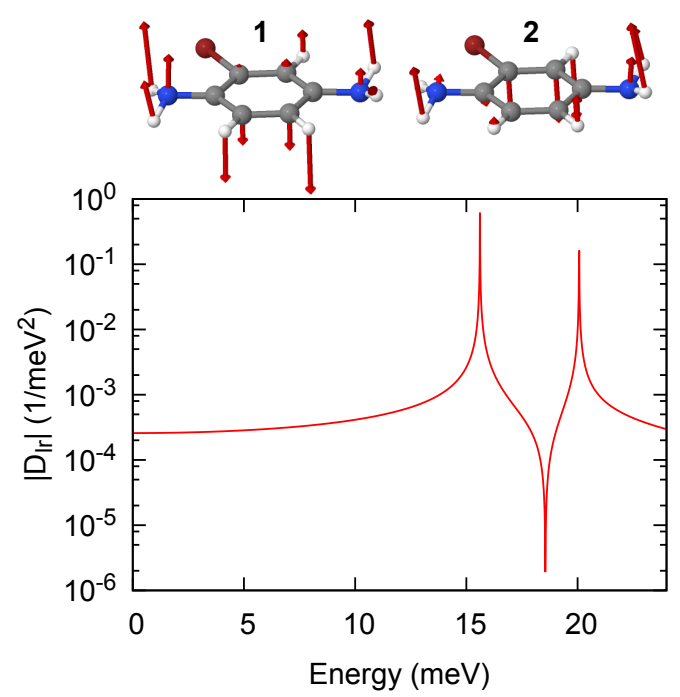

FIG. 3. In the upper part we visualize the two modes 1 and 2 that are responsible for the destructive interference feature at $19 \mathrm{meV}$ in the benzenediamine molecule, substituted with $X=\mathrm{Br}$. The first mode is at an energy of $15.61 \mathrm{meV}$ and the second one at $20.07 \mathrm{meV}$. Since for both modes the arrows on the $\mathrm{N}$ atoms on the left and right sides point in the same direction, they interfere destructively. The graph below shows the calculated absolute value of the relevant Green's function element of the isolated molecule, taking into account in Eq. (4) only the modes 1 and 2 and using $\eta=10^{-3} \mathrm{meV}$.

investigate the so-called cumulative thermal conductance

$$
\kappa_{\mathrm{pn}}^{\mathrm{c}}(E, T)=\frac{1}{h} \int_{0}^{E} d E^{\prime} E^{\prime} \tau_{\mathrm{pn}}\left(E^{\prime}\right) \frac{\partial n\left(E^{\prime}, T\right)}{\partial T}
$$

that is defined as the thermal conductance due to phonon modes up to a given energy $E$. We show in the inset of Fig. 2(b) the room-temperature cumulative thermal conductance as a function of energy for the $\mathrm{Au}$ benzenediamine-Au junctions with $X=\mathrm{H}, \mathrm{Br}$, I. As one can see, the introduction of substituents actually modifies the phonon transport at all energies, but the change is particularly drastic at the energies at which the antiresonances occur. This illustrates that the destructive interference effects, induced by substituent atoms, play a key role in the reduction of the heat transport and can be used as a strategy to modify the thermal conductance.

In order to understand the origin of the destructive interference effect, discussed above for $\mathrm{Br}$ and I, we follow an argument based on the symmetry of the molecular orbitals developed for electron transport ${ }^{33}$. Since the structure of the transport formalism is almost identical for electrons and phonons, with the only difference arising from the equations of motion, this argument can be straightforwardly adapted to phonon transport. Assuming for simplicity only nearest neighbor couplings, the idea is as follows. If the molecule is attached to the left lead at position 1 with coupling constant $k_{\mathrm{IL}}$ and at position $\mathrm{r}$ with coupling constant $k_{\mathrm{rR}}$ to the right lead, then 
the transmission function can be expressed as

$$
\tau_{\mathrm{pn}}(E)=\frac{\pi^{2}}{E^{2}} k_{\mathrm{lL}}^{2} k_{\mathrm{rR}}^{2} \rho_{\mathrm{L}}(E) \rho_{\mathrm{R}}(E)\left|D_{\mathrm{lr}}(E)\right|^{2} .
$$

Here $\rho_{\alpha}(E)$ is the local density of states at the lead atom $\alpha=\mathrm{L}, \mathrm{R}$ that is connected to the atom $\mathrm{l}, \mathrm{r}$ of the molecule, respectively, and $D_{\operatorname{lr}}(E)$ is the lr-matrix element of the Green's function at energy $E$. Let us assume that the typical embedding self-energies $\Pi_{\alpha}(E)$, which describe the coupling of the central junction part to the left and right electrodes ${ }^{55,56}$ and appear in the full expression for $D_{\operatorname{lr}}(E)$, can be neglected, which is for instance the case if molecule-lead couplings are not too strong. In this situation the Green's function entering in the previous equation can be approximated by the corresponding Green's function of the isolated molecule. It is given by the following spectral representation

$$
D_{\mathrm{lr}}(E)=\sum_{j} \frac{C_{\mathrm{l} j} C_{\mathrm{r} j}^{*}}{(E+i \eta)^{2}-E_{j}^{2}},
$$

where $C_{n j}$ is the $n$-th component of the $j$-th eigenfunction or vibrational mode. Given the dynamical matrix $\boldsymbol{K}$, the eigenfunctions $C_{n j}$ and angular momentum frequencies $\omega_{j}$ are obtained by solving the secular equation

$$
\boldsymbol{K} \boldsymbol{C}_{j}=\omega_{j}^{2} \boldsymbol{C}_{j}
$$

In the spectral representation in Eq. (4), $\eta$ is a small imaginary part that prevents $D_{\operatorname{lr}}(E)$ from diverging at $E \rightarrow E_{j}$, and $E_{j}=\hbar \omega_{j}$ is the energy of the $j$-th vibrational mode of the isolated molecule. As discussed in Ref. [33] for molecular junctions and in Ref. [66] for mesoscopic systems, a destructive interference occurs between vibrational modes $j$ that exhibit the same sign of the product $C_{\mathrm{l} j} C_{\mathrm{r} j}^{*}$, or more pictorially, the same parity of vibrational modes at the lead-connecting molecular sites. This conclusion is obvious from the general form of the Green's function in Eq. (4). If the embedding selfenergies $\Pi_{\alpha}(E)$ are taken into account, they may lead to renormalizations of level positions and hence antiresonance peaks. They are also important to describe level broadenings, which are mimicked in Eq. (4) by $\eta$, and hence to avoid any divergencies of the transmission in Eq. (3) at resonance positions $E=E_{j}$.

Let us now apply these ideas to understand the antiresonance that appears at around $19 \mathrm{meV}$ in the transmission function for the benzenediamine junction with the substituent $X=\mathrm{Br}$, see Fig. 2(a). For this purpose we first analyzed the vibrational modes of the isolated molecule within DFT and identified the two modes at $15.61 \mathrm{meV}$ and $20.07 \mathrm{meV}$, which are shown in the upper part of Fig. 3. Taking into account only these two modes in the sum over $j$ in Eq. (4), we display in this figure also the corresponding absolute value of $D_{\mathrm{lr}}(E)$. Here the connection to the leads has been assumed to be established only at the $\mathrm{N}$ atoms and the coefficients $C_{n j}$ have been taken from the calculations of the isolated molecule.
Since these two modes exhibit the same oscillation direction on the $\mathrm{N}$ atoms, the products $C_{11} C_{\mathrm{r} 1}^{*}$ and $C_{12} C_{\mathrm{r} 2}^{*}$ have the same sign, and the modes 1 and 2 are expected to interfere destructively. This is confirmed by the behavior of $\left|D_{\operatorname{lr}}(E)\right|$ in Fig. 3, which exhibits two peaks at the energies of the two modes and a minimum at around $19 \mathrm{meV}$, energetically located between these two modes. The position of the minimum is in good agreement with the minimum in the transmission curve in Fig. 2. Let us mention that the same considerations apply for the molecule with $X=\mathrm{I}$, where we find two modes of similar characteristics at energies around 14.7 and $19.5 \mathrm{meV}$.

One may wonder whether the antiresonances, resulting from destructive interference, survive upon the elongation of the molecular junctions. We have investigated this aspect for the junctions with the benzene derivatives studied in Fig. 2 and found that the appearance of antiresonances is quite robust against stretching or compression. This is indeed expected since, as we have discussed above, the antiresonances originate from the interference of internal vibrational modes of the molecules, and they are therefore not greatly affected by variations of the distance between the electrodes.

Following the analogy with electronic systems, we analyze now, how the inclusion of additional substituents modifies the interference patterns ${ }^{36}$. For this purpose we investigate the phonon transport through benzenediamine junctions, if two $\mathrm{H}$ atoms are replaced by two $\mathrm{Br}$ atoms. Moreover we study the influence of the exact position, where these $\mathrm{Br}$ atoms are incorporated, and examine the three molecular junctions shown in the upper part of Fig. 4. All vibrational modes between 15 and 20 $\mathrm{meV}$ in the central region of the corresponding junctions are shown in Fig. 5.

The results for the phonon transmission are displayed in Fig. 4(a). As one can see, for molecule 1 a destructive interference antiresonance appears at about $17 \mathrm{meV}$. The origin of this feature is, as for the previous compounds, the interference between two modes that lie close in energy. These are the modes with energies of 17.75 and $18.95 \mathrm{meV}$ in the left column of Fig. 5 that show the same parity on the terminal $\mathrm{N}$ atoms of the molecule. The difference with respect to the singly-substituted molecules discussed above is that these modes have no analogues in the isolated molecule. Instead they are hybrid modes in the sense that they also involve vibrations of the gold atoms in the electrodes. Additionally, a reduced transmission peak at $12.5 \mathrm{meV}$ arises, which is due to a localized mode that is asymmetrically coupled to the electrodes. Interestingly, the other two molecules 2 and 3 do not exhibit any pronounced antiresonance, resulting from destructive interference, see Fig. 4(a), which we attribute to the lack of the necessary symmetry of the vibrational modes on the terminal $\mathrm{N}$ atoms, see Fig. 5. This shows that not only the masses of the substituents play a role, but also the exact position, where they are introduced. The changes in the transmission are reflected in the corresponding thermal conductance results. As we see in 

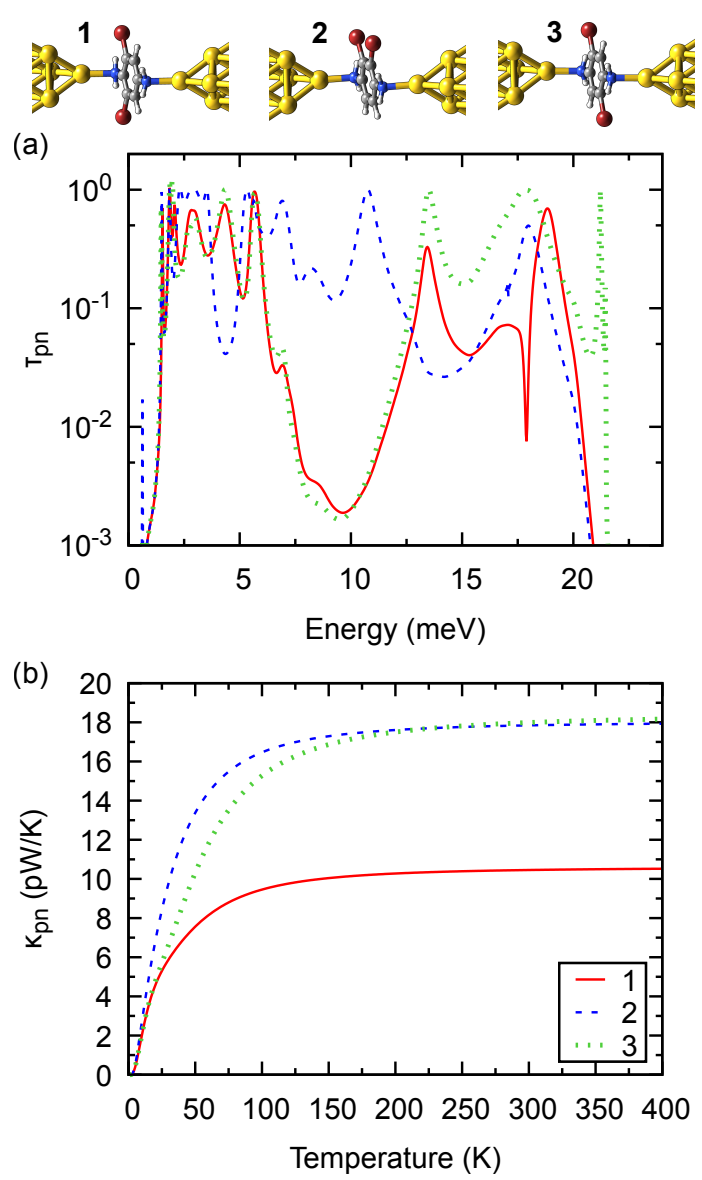

FIG. 4. (a) Phonon transmission as a function of energy for the three molecular junctions shown above this panel, where two $\mathrm{H}$ atoms of the benzenediamine molecule have been replaced by two $\mathrm{Br}$ atoms. (b) The corresponding temperature dependence of the phononic thermal conductance. The legend in panel (b) applies also to panel (a).

Fig. 4(b), while molecule 1 exhibits a largely reduced thermal conductance at room temperature as compared to both the singly substituted case and the unsubstituted benzenediamine, molecules 2 and 3 exhibit conductance values that are similar to those of the singly substituted case.

Now we show that the basic concepts discussed above also apply to other, more complex molecules. For this purpose, we consider OPE3. This molecule has been analyzed in the context of phonon transport in Ref. [49] with the help of semi-empirical methods and considering Si as well as graphene-nanoribbon electrodes. First we discuss the influence of the binding configuration, by examining para- vs. meta-OPE3, as shown in the upper part of Fig. 6. In this case our ab initio results for the phonon transmission, which are displayed in Fig. 6(a), show that for meta-OPE3 several resonance peaks at energies below $15 \mathrm{meV}$ appear shifted to lower energies as compared to para-OPE3, while there is a pronounced decrease of the transmission for the meta-OPE3 above. The strong sup-
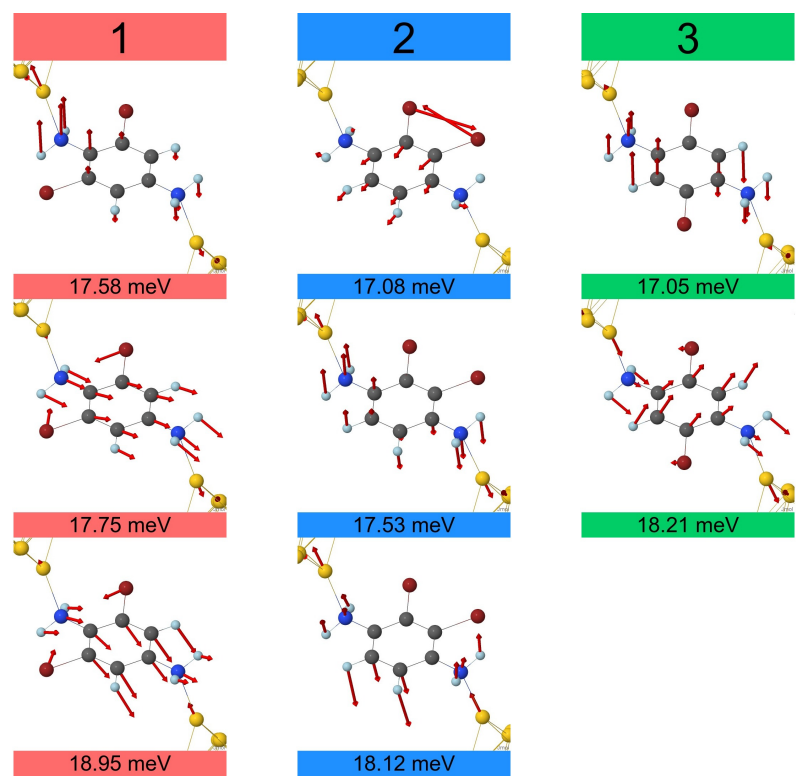

FIG. 5. All vibrational modes in the central region of the three molecular junctions discussed in Fig. 4 in the energy range between 15 and $20 \mathrm{meV}$.

pression of the transmission at around $18 \mathrm{meV}$ is due to an interference effect, in which two quasi-degenerate modes are involved, as explained in Ref. [49]. In total the meta-OPE3 thermal conductance is reduced by a factor of 2 , as compared to para-OPE3.

As in the case of benzene, the thermal conductance of $\mathrm{Au}-\mathrm{OPE} 3-\mathrm{Au}$ junctions can be tuned by substituting one $\mathrm{H}$ atom in the central benzene ring with a halogen atom, as sketched in the upper part of Fig. 7. Similar to benzenediamine in Fig. 2, one can see in Fig. 7(a) that the phonon transmission exhibits an antiresonance at energies around 15-20 meV for the substituents $\mathrm{Br}$ and I, but now this feature also appears for $\mathrm{Cl}$. Again the occurrence of destructive interferences below the Debye energy of gold leads to a suppression of the corresponding thermal conductance, as we show in Fig. 7(b). We find a monotonically decreasing thermal conductance with increasing mass of the substituent. As in the case of the benzene derivatives this behavior is due to the fact that, upon introducing the substituents, the energies of the vibrational modes decrease and the antiresonances, resulting from the interference effects, are redshifted at the same time. Thus, since the contribution of a vibrational mode scales with its energy, see Eq. (1), $\kappa_{\text {pn }}$ is reduced upon increasing mass of the substituents.

Let us mention that we are not aware that the exact OPE3 compounds, shown in Fig. 7, have been synthesized. But closely related molecules exist, in which the central ring of an OPE3-diamine has been modified, among others, by the inclusion of several fluorine atoms and which have been studied in the context of singlemolecule junction experiments ${ }^{67}$. So, we think that there should not be any fundamental problem to synthesize the 


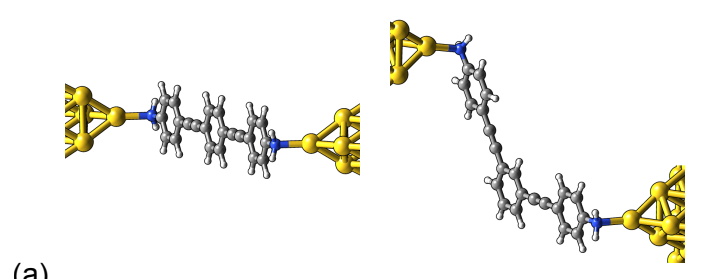

(a)
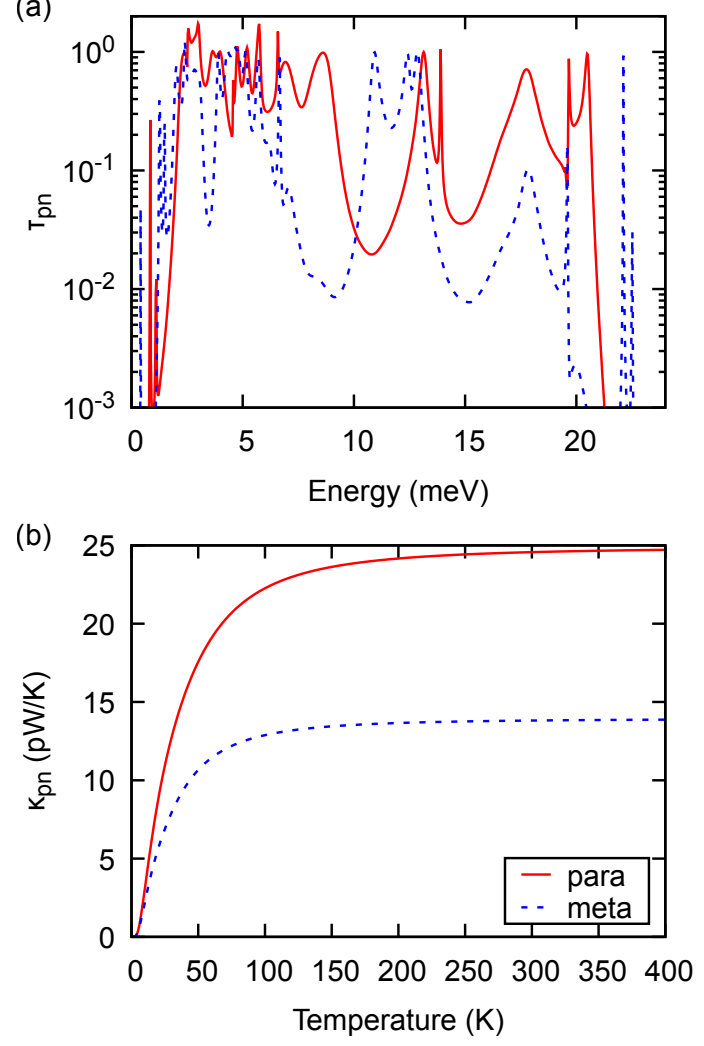

FIG. 6. (a) Phonon transmission as a function of energy for the Au-OPE3-Au junctions shown above this panel for both para (left) and meta (right) binding configurations at the central benzene ring. (b) The corresponding phononic thermal conductance as a function of temperature. The legend in panel (b) applies also to panel (a).

compounds discussed in this work. In this respect, it is also worth stressing that our DFT calculations demonstrate that these molecules are indeed stable

To conclude the discussion of our results on phonon heat transport, it is important to emphasize that in all the molecular junctions investigated in this work, the room-temperature thermal conductance is dominated by the contribution of phonons. Therefore the impact of the interference effects discussed here should be, in principle, amenable to measurement. This point is discussed in detail in Appendix A, where we present our results for the electronic contribution to the thermal conductance.
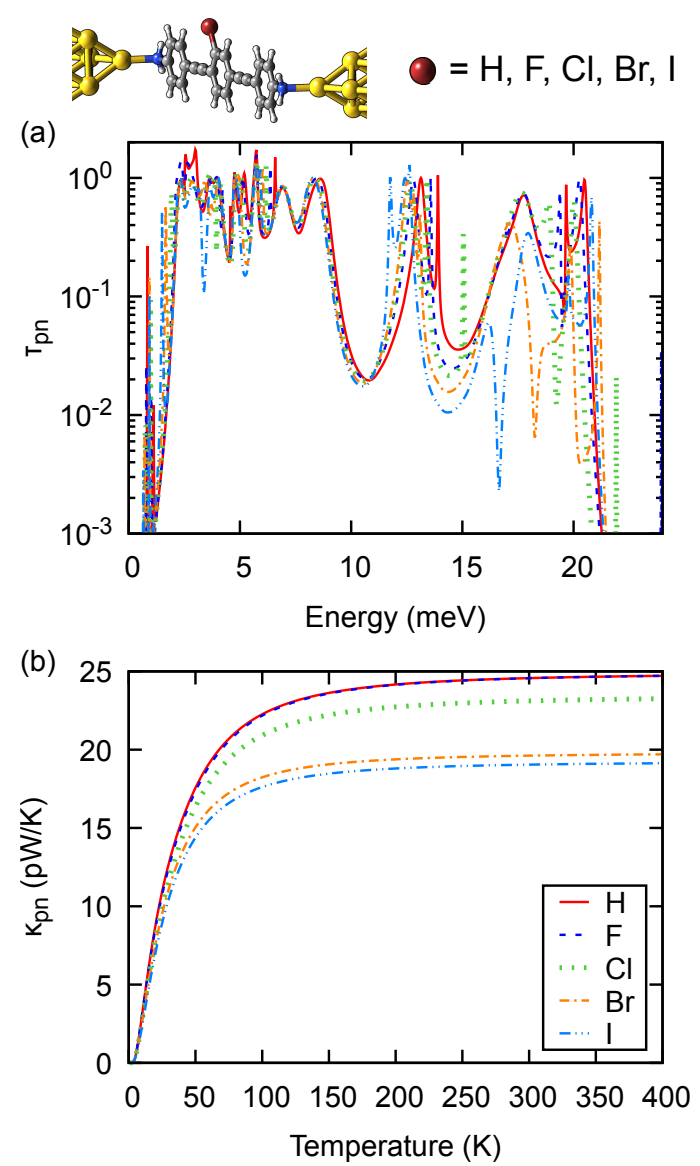

FIG. 7. (a) Phonon transmission as a function of energy for $\mathrm{Au}$-para-OPE3-Au junctions, where a $\mathrm{H}$ atom of the central benzene ring has been substituted by a halogen atom $(X=\mathrm{F}$, $\mathrm{Cl}, \mathrm{Br}, \mathrm{I}$ ), see plot above this panel. (b) The corresponding phononic thermal conductance as a function of temperature for the different para-OPE3 derivatives. The legend in panel (b) applies also to panel (a).

\section{CONCLUSIONS}

Making use of a full $a b$ initio transport method, we have analyzed the influence of phonon interference on the thermal conductance of benzene and OPE3 derivatives attached to $\mathrm{Au}$ electrodes via amino groups. We have found that for unsubstituted benzene no interference effects are visible, irrespective of the binding configuration, which is due to the small Debye energy of gold. We have also shown that by substituting one $\mathrm{H}$ atom with a halogen atom of increasing mass leads to the appearance of Fano-like resonances at energies below the $\mathrm{Au}$ Debye energy, which in turn leads to a reduction of thermal conductances by up to a factor of 1.7 . We were able to relate this kind of antiresonance feature to the destructive interference between two modes of the free molecule with the same symmetry in the region, where the molecule is connected to the leads. We have also shown that the thermal conductance can be further re- 
duced by increasing the number of substituent atoms and arranging them in appropriate positions.

Finally, we have used similar concepts to tune the thermal conductance of junctions based on OPE3 molecules. In particular, we have demonstrated that the thermal conductance of meta-OPE3 junctions is clearly reduced as compared to the para-OPE3 case mainly due to an interference between two quasi-degenerate vibrational modes. On the other hand, we have also shown that the thermal conductance of a linear Au-OPE3-Au junction can be reduced by incorporating halogen atoms in the central ring of this molecule. Again, this effect is due to the appearance of destructive phonon interference.

In summary, we have demonstrated that, in analogy to the electronic transport, the thermal conductance of molecular junctions can be controlled by means of phonon interference effects. Even if the effect for phonons is not as dramatic as for the electronic analog, our findings are important to achieve heat management in nanostructured metal-molecule hybrid systems and to explore their potential in thermoelectric applications.

\section{ACKNOWLEDGMENT}

J.C.K. thanks A. Irmler for stimulating discussions. J.C.K. and F.P. gratefully acknowledge funding from the Carl Zeiss foundation and the Junior Professorship Program of the Ministry of Science, Research, and the Arts of the state of Baden Württemberg. J.C.C. is supported by the German Research Foundation (DFG) and the Collaborative Research Center (SFB) 767, which sponsor his stay at the University of Konstanz as Mercator Fellow, as well as the Spanish Ministry of Economy and Competitiveness (Contract No. FIS2014-53488-P). An important part of the numerical modeling was carried out on the computational resources of the bwHPC program, namely the bwUniCluster and the JUSTUS HPC facility.

\section{Appendix A: Electronic thermal conductance}

In this appendix we briefly discuss our results on the electronic contribution to the thermal conductance for the different molecular junctions investigated in this work. The goal is to show that in these junctions the thermal transport is dominated by phonons and that the interference effects, predicted here, should therefore be observable experimentally. We neglect contributions to the thermal conductance from near field radiative heat transfer, whose significance can be controlled by the macroscopic shape of the electrodes ${ }^{57}$.

As in the phononic case we assume that the electronic transport is dominated by elastic tunneling processes. Thus, we compute the electronic contribution to the thermal conductance in the linear response regime within the
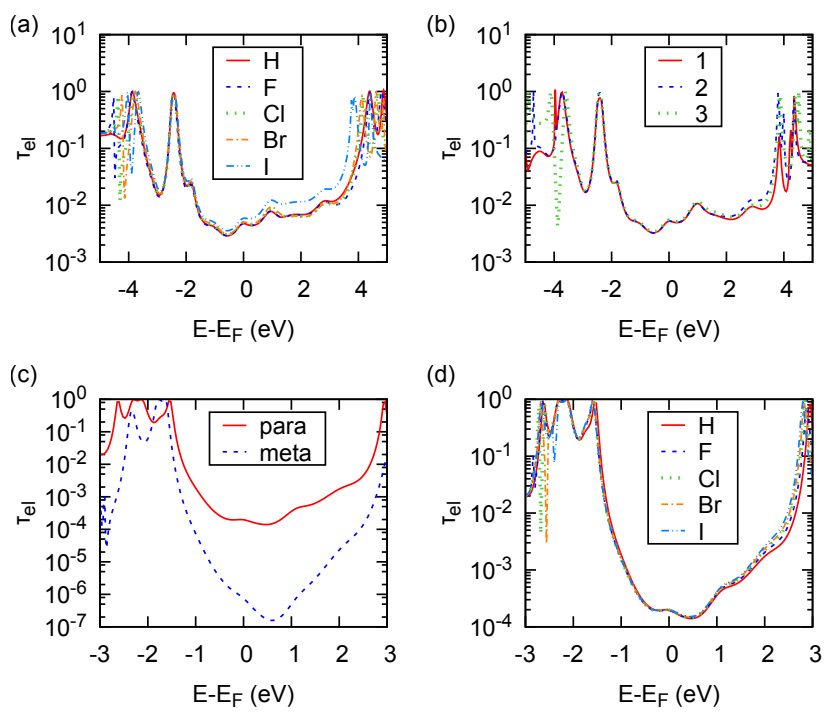

FIG. 8. Electronic transmission as a function of energy, computed for (a) the molecular junctions of Fig. 2 based on benzene with a single halogen atom as substituent, (b) the junctions of Fig. 4 based on benzene with two $\mathrm{Br}$ atoms as substituents, (c) the para- and meta-bonded OPE3 junctions of Fig. 6 and (d) the OPE3-based junctions of Fig. 7 with a single substituent on the central benzene ring.

Landauer-Büttiker formalism. It is given by ${ }^{10}$

$$
\kappa_{\mathrm{el}}(T)=\frac{2}{h T}\left(K_{2}(T)-\frac{K_{1}(T)^{2}}{K_{0}(T)}\right),
$$

where the coefficients are defined as

$$
K_{n}(T)=\int_{-\infty}^{\infty} d E \tau_{\mathrm{el}}(E)\left(-\frac{\partial f(E, T)}{\partial E}\right)(E-\mu)^{n},
$$

$\tau_{\mathrm{el}}(E)$ is the energy-dependent electron transmission, and $f(E, T)=\left\{\exp \left[(E-\mu) / k_{\mathrm{B}} T\right]+1\right\}^{-1}$ is the Fermi function. Here, the chemical potential $\mu \approx E_{\mathrm{F}}$ is approximately given by the Fermi energy $E_{\mathrm{F}}$ of the $\mathrm{Au}$ electrodes.

To compute the electronic transmission function, we have employed an approach based on the combination of DFT and nonequilibrium Green's function techniques that we have interfaced to TURBOMOLE and explained in detail in Ref. [68]. Moreover, in order to correct for the known inaccuracies in DFT related to quasiparticle energies, we have made use of the DFT $+\Sigma$ approach $^{65}$, which was implemented in our quantum transport method as described in Ref. [69].

In Fig. 8 we show our results for the electronic transmission $\tau_{\mathrm{el}}$ as a function of energy for all the junctions of Figs. 2, 4, 6, and 7 based on both benzene and OPE3 derivatives. In all cases the electronic transport proceeds mainly through the tail of the HOMO of the molecule in an off-resonant situation. The results for the transport properties of these molecular junctions at room temperature are summarized in Table I. We present there both 
TABLE I. Computed room-temperature phononic thermal conductance $\kappa_{\mathrm{pn}}$, electronic thermal conductance $\kappa_{\mathrm{el}}$ and electrical conductance $G$ in units of the electrical conductance quantum $G_{0}=2 e^{2} / h$ for the different molecular junctions investigated in this work. The last column shows, when available, the experimental value of the electrical conductance, as obtained from the peaks of conductance histograms. Experimental uncertainties due to broad distributions of conductance values have been omitted.

\begin{tabular}{lcccc}
\hline Molecule & $\kappa_{\mathrm{pn}}(\mathrm{pW} / \mathrm{K})$ & $\kappa_{\mathrm{el}}(\mathrm{pW} / \mathrm{K})$ & $G\left(G_{0}\right)($ theory $)$ & $G\left(G_{0}\right)($ exp. $)$ \\
\hline 1,4-diaminobenzene (para) & 25.24 & 2.61 & $4.7 \times 10^{-3}$ & $6.4 \times 10^{-3}[70]$ \\
2-fluoro-1,4-diaminobenzene & 24.40 & 2.62 & $4.7 \times 10^{-3}$ & $5.8 \times 10^{-3}[70]$ \\
2-chloro-1,4-diaminobenzene & 22.17 & 2.70 & $4.9 \times 10^{-3}$ & $6.0 \times 10^{-3}[70]$ \\
2-bromo-1,4-diaminobenzene & 16.94 & 2.78 & $5.0 \times 10^{-3}$ & $6.1 \times 10^{-3}[70]$ \\
2-iodo-1,4-diaminobenzene & 15.24 & 3.25 & $5.8 \times 10^{-3}$ & \\
2,5-dibromo-1,4-diaminobenzene & 17.85 & 2.88 & $5.20 \times 10^{-3}$ & \\
2,6-dibromo-1,4-diaminobenzene & 10.46 & 2.88 & $5.19 \times 10^{-3}$ & \\
2,3-dibromo-1,4-diaminobenzene & 17.99 & 3.02 & $5.44 \times 10^{-3}$ & \\
OPE3 (para) & 24.57 & 0.11 & $1.94 \times 10^{-4}$ & $2.6 \times 10^{-5}[71], 1.27 \times 10^{-4}[72]$ \\
OPE3 (meta) & 13.82 & $4.24 \times 10^{-4}$ & $7.53 \times 10^{-7}$ & \\
F-OPE3 & 24.56 & 0.11 & $1.93 \times 10^{-4}$ & \\
Cl-OPE3 & 23.12 & 0.11 & $1.96 \times 10^{-4}$ & \\
Br-OPE3 & 19.62 & 0.11 & $1.97 \times 10^{-4}$ & \\
I-OPE3 & 19.04 & 0.11 & $2.00 \times 10^{-4}$ & \\
\hline
\end{tabular}

the phononic and electronic thermal conductances $\kappa_{\text {pn }}$ and $\kappa_{\mathrm{el}}$, as well as the electrical conductance

$$
G(T)=G_{0} K_{0}(T)
$$

with $G_{0}=2 e^{2} / h$. Moreover, when available, we also report the experimental values for $G$. The electric conductance can be used together with the WiedemannFranz law $\kappa_{e l} \approx L_{0} G T$ with the Lorentz number $L_{0}=$ $\left(k_{\mathrm{B}} / e\right)^{2} \pi^{2} / 3$ to estimate the electronic thermal conductance. We find the Wiedemann-Franz law to be approximately fulfilled for our computed molecular junctions, and the comparison of experimental and theoretical electrical conductance values $G$ can hence be used to estimate uncertainties in the theoretical $\kappa_{\mathrm{el}}$.

The key point is that in all the studied cases the electronic thermal conductance is considerably smaller than the corresponding phononic thermal conductance. In particular, for the junctions based on the benzene derivatives the electronic contribution is at least 5 times smaller than the phononic one, while for the OPE3 compounds the electronic contribution is more than two orders of magnitude smaller. Due to their lower $G$ the longer molecules are thus advantageous, if we want to exclude the contribution of electrons to heat transport.

Moreover, in order to illustrate that phonons dominate the thermal transport in these junctions for a wide temperature range, we show in Fig. 9 the temperature dependence of the phononic and electronic thermal conductances for the Au-1,4-diaminobenzene- $\mathrm{Au}$ and $\mathrm{Au}-2$ bromo-1,4-diaminobenzene-Au junctions of Fig. 2. As one can see, the phononic contribution largely dominates at all temperatures between 10 and 400 K. Similar results hold for all the other molecular junctions.

To conclude, the results presented in this appendix confirm that the thermal transport in the molecular junctions studied in this work is dominated by phonons. The predicted interference effects should therefore be visible in possible experiments.
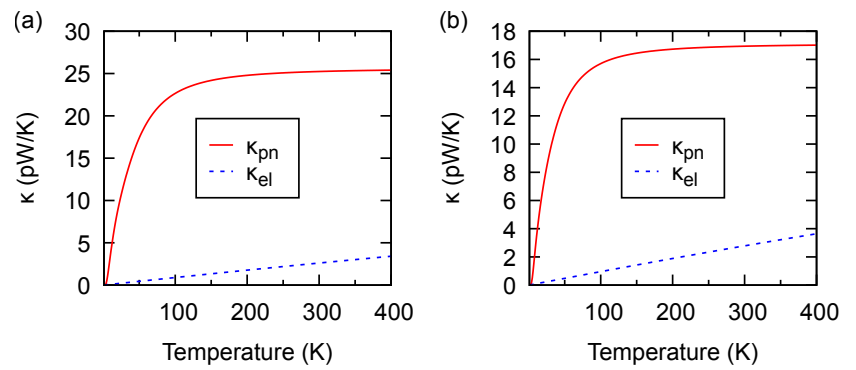

FIG. 9. Temperature dependence of the phononic and electronic contribution to the thermal conductance for the (a) Au-1,4-diaminobenzene-Au and (b) Au-2-bromo-1,4diaminobenzene-Au junctions of Fig. 2. 
* Jan.Kloeckner@uni-konstanz.de

1 D. G. Cahill, P. V. Braun, G. Chen, D. R. Clarke, S. Fan, K. E. Goodson, P. Keblinski, W. P. King, G. D. Mahan, A. Majumdar, H. J. Maris, S. R. Phillpot, E. Pop, and L. Shi, Appl. Phys. Rev. 1, 011305 (2014).

2 M. Maldovan, Nat. Mater. 14, 667 (2015).

3 M. N. Luckyanova, J. Garg, K. Esfarjani, A. Jandl, M. T. Bulsara, A. J. Schmidt, A. J. Minnich, S. Chen, M. S. Dresselhaus, Z. Ren, E. A. Fitzgerald, and G. Chen, Science 338, 936 (2012).

4 J. Ravichandran, A. K. Yadav, R. Cheaito, P. B. Rossen, A. Soukiassian, S. J. Suresha, J. C. Duda, B. M. Foley, C.-H. Lee, Y. Zhu, A. W. Lichtenberger, J. E. Moore, D. A. Muller, D. G. Schlom, P. E. Hopkins, A. Majumdar, R. Ramesh, and M. A. Zurbuchen, Nat. Mater. 13, 168 (2014).

${ }^{5}$ H. Han, L. G. Potyomina, A. A. Darinskii, S. Volz, and Y. A. Kosevich, Phys. Rev. B 89, 180301 (2014).

6 U. Fano, Phys. Rev. 124, 1866 (1961).

7 A. E. Miroshnichenko, S. Flach, and Y. S. Kivshar, Rev. Mod. Phys. 82, 2257 (2010).

8 B. Luk'yanchuk, N. I. Zheludev, S. A. Maier, N. J. Halas, P. Nordlander, H. Giessen, and C. T. Chong, Nat. Mater. 9, 707 (2010).

9 P. Fan, Z. Yu, S. Fan, and M. L. Brongersma, Nat. Mater. 13, 471 (2014).

10 J. C. Cuevas and E. Scheer, Molecular Electronics: An Introduction to Theory and Experiment, 2nd ed. (World Scientific, Singapore, 2017).

11 P. Sautet and C. Joachim, Chem. Phys. Lett. 153, 511 (1988).

12 E. G. Emberly and G. Kirczenow, Phys. Rev. B 58, 10911 (1998).

13 R. Baer and D. Neuhauser, J. Am. Chem. Soc. 124, 4200 (2002).

14 R. Stadler, M. Forshaw, and C. Joachim, Nanotechnology 14, 138 (2003).

15 D. Walter, D. Neuhauser, and R. Baer, Chem. Phys. 299, 139 (2004).

16 D. M. Cardamone, C. A. Stafford, and S. Mazumdar, Nano Lett. 6, 2422 (2006).

17 C. A. Stafford, D. M. Cardamone, and S. Mazumdar, Nanotechnology 18, 424014 (2007).

18 S.-H. Ke, W. Yang, and H. U. Baranger, Nano Lett. 8, 3257 (2008).

19 D. Q. Andrews, G. C. Solomon, R. H. Goldsmith, T. Hansen, M. R. Wasielewski, R. P. V. Duyne, and M. A. Ratner, J. Phys. Chem. C 112, 16991 (2008).

${ }^{20}$ G. C. Solomon, D. Q. Andrews, T. Hansen, R. H. Goldsmith, M. R. Wasielewski, R. P. V. Duyne, and M. A. Ratner, J. Chem. Phys. 129, 054701 (2008).

${ }^{21}$ G. C. Solomon, D. Q. Andrews, R. H. Goldsmith, T. Hansen, M. R. Wasielewski, R. P. Van Duyne, and M. A. Ratner, J. Am. Chem. Soc. 130, 17301 (2008).

22 D. Q. Andrews, G. C. Solomon, R. P. Van Duyne, and M. A. Ratner, J. Am. Chem. Soc. 130, 17309 (2008).

23 R. Stadler, Phys. Rev. B 80, 125401 (2009).

24 G. C. Solomon, C. Herrmann, T. Hansen, V. Mujica, and M. A. Ratner, Nat. Chem. 2, 223 (2010).

25 T. Markussen, J. Schiøtz, and K. S. Thygesen, J. Chem. Phys. 132, 224104 (2010).
${ }^{26}$ G. Géranton, C. Seiler, A. Bagrets, L. Venkataraman, and F. Evers, J. Chem. Phys. 139, 234701 (2013).

27 L. A. Zotti, E. Leary, M. Soriano, J. C. Cuevas, and J. J. Palacios, J. Am. Chem. Soc. 135, 2052 (2013).

28 A. Grigoriev, J. Sköldberg, G. Wendin, and Ž. Crljen, Phys. Rev. B 74, 045401 (2006).

29 T. A. Papadopoulos, I. M. Grace, and C. J. Lambert, Phys. Rev. B 74, 193306 (2006).

${ }^{30}$ M. Ernzerhof, J. Chem. Phys. 127, 204709 (2007).

31 X. Shi, Z. Dai, and Z. Zeng, Phys. Rev. B 76, 235412 (2007).

32 C. M. Finch, V. M. García-Suárez, and C. J. Lambert, Phys. Rev. B 79, 033405 (2009).

33 K. Yoshizawa, T. Tada, and A. Staykov, J. Am. Chem. Soc. 130, 9406 (2008).

34 T. Markussen, R. Stadler, and K. S. Thygesen, Nano Lett. 10, 4260 (2010).

${ }^{35}$ K. G. L. Pedersen, M. Strange, M. Leijnse, P. Hedegård, G. C. Solomon, and J. Paaske, Phys. Rev. B 90, 125413 (2014).

36 M. H. Garner, G. C. Solomon, and M. Strange, J. Phys. Chem. C 120, 9097 (2016).

37 M. Mayor, H. B. Weber, J. Reichert, M. Elbing, C. von Hänisch, D. Beckmann, and M. Fischer, Angew. Chem., Int. Ed. 42, 5834 (2003).

38 D. Fracasso, H. Valkenier, J. C. Hummelen, G. C. Solomon, and R. C. Chiechi, J. Am. Chem. Soc. 133, 9556 (2011).

39 W. Hong, H. Li, S.-X. Liu, Y. Fu, J. Li, V. Kaliginedi, S. Decurtins, and T. Wandlowski, J. Am. Chem. Soc. 134, 19425 (2012).

40 C. M. Guedon, H. Valkenier, T. Markussen, K. S. Thygesen, J. C. Hummelen, and S. J. van der Molen, Nat. Nanotech. 7, 305 (2012).

41 S. V. Aradhya, J. S. Meisner, M. Krikorian, S. Ahn, R. Parameswaran, M. L. Steigerwald, C. Nuckolls, and L. Venkataraman, Nano Lett. 12, 1643 (2012).

${ }^{42}$ H. Vazquez, R. Skouta, S. Schneebeli, M. Kamenetska, R. Breslow, L. Venkataraman, and M. Hybertsen, Nat. Nanotech. 7, 663 (2012).

43 V. Rabache, J. Chaste, P. Petit, M. L. Della Rocca, P. Martin, J.-C. Lacroix, R. L. McCreery, and P. Lafarge, J. Am. Chem. Soc. 135, 10218 (2013).

44 C. R. Arroyo, S. Tarkuc, R. Frisenda, J. S. Seldenthuis, C. H. M. Woerde, R. Eelkema, F. C. Grozema, and H. S. J. van der Zant, Angew. Chem., Int. Ed. 52, 3152 (2013).

45 C. R. Arroyo, R. Frisenda, K. Moth-Poulsen, J. S. Seldenthuis, T. Bjørnholm, and H. S. J. van der Zant, Nanoscale Res. Lett. 8, 234 (2013).

46 J. Xia, B. Capozzi, S. Wei, M. Strange, A. Batra, J. R. Moreno, R. J. Amir, E. Amir, G. C. Solomon, L. Venkataraman, and L. M. Campos, Nano Lett. 14, 2941 (2014).

47 D. Z. Manrique, C. Huang, M. Baghernejad, X. Zhao, O. A. Al-Owaedi, H. Sadeghi, V. Kaliginedi, W. Hong, M. Gulcur, T. Wandlowski, M. R. Bryce, and C. J. Lambert, 6, 6389 (2015).

48 R. Frisenda, V. A. E. C. Janssen, F. C. Grozema, H. S. J. van der Zant, and N. Renaud, Nat. Chem. 8, 1099 (2016).

49 T. Markussen, J. Chem. Phys. 139, 244101 (2013).

50 M. Famili, I. Grace, H. Sadeghi, and C. J. Lambert, Chem. Phys. Chem. 18, 1234 (2017). 
51 Q. Li, I. Duchemin, S. Xiong, G. C. Solomon, and D. Donadio, J. Phys. Chem. C 119, 24636 (2015).

${ }^{52}$ L. Cui, W. Jeong, S. Hur, M. Matt, J. C. Klöckner, F. Pauly, P. Nielaba, J. C. Cuevas, E. Meyhofer, and P. Reddy, Science 355, 1192 (2017).

53 N. Mosso, U. Drechsler, F. Menges, P. Nirmalraj, S. Karg, H. Riel, and B. Gotsmann, Nat. Nanotech. 12, 430 (2017).

54 A. Jain and A. J. H. McGaughey, Phys. Rev. B 93, 081206 (2016).

55 M. Bürkle, T. J. Hellmuth, F. Pauly, and Y. Asai, Phys. Rev. B 91, 165419 (2015).

56 J. C. Klöckner, M. Bürkle, J. C. Cuevas, and F. Pauly, Phys. Rev. B 94, 205425 (2016).

57 J. C. Klöckner, R. Siebler, J. C. Cuevas, and F. Pauly, Phys. Rev. B 95, 245404 (2017).

58 TURBOMOLE GmbH Karlsruhe, TURBOMOLE, http://www.turbomole.com. TURBOMOLE was a development of the University of Karlsruhe and Forschungszentrum Karlsruhe from 1989 to 2007, and development has been undertaken by TURBOMOLE GmbH since 2007.

${ }^{59}$ P. Deglmann, F. Furche, and R. Ahlrichs, Chem. Phys. Lett. 362, 511 (2002).

60 P. Deglmann, K. May, F. Furche, and R. Ahlrichs, Chem. Phys. Lett. 384, 103 (2004).

61 J. P. Perdew and Y. Wang, Phys. Rev. B 45, 13244 (1992).

62 J. P. Perdew, K. Burke, and M. Ernzerhof, Phys. Rev.
Lett. 77, 3865 (1996)

63 F. Weigend and R. Ahlrichs, Phys. Chem. Chem. Phys. 7, 3297 (2005).

${ }^{64}$ F. Weigend, Phys. Chem. Chem. Phys. 8, 1057 (2006).

65 S. Y. Quek, L. Venkataraman, H. J. Choi, S. G. Louie, M. S. Hybertsen, and J. B. Neaton, Nano Lett. 7, 3477 (2007).

66 H.-W. Lee, Phys. Rev. Lett. 82, 2358 (1999).

67 M. T. González, X. Zhao, D. Z. Manrique, D. Miguel, E. Leary, M. Gulcur, A. S. Batsanov, G. Rubio-Bollinger, C. J. Lambert, M. R. Bryce, and N. Agraï, J. Phys. Chem. C 118, 21655 (2014).

68 F. Pauly, J. K. Viljas, U. Huniar, M. Häfner, S. Wohlthat, M. Bürkle, J. C. Cuevas, and G. Schön, New J. Phys. 10, 125019 (2008).

${ }^{69}$ L. A. Zotti, M. Bürkle, F. Pauly, W. Lee, K. Kim, W. Jeong, Y. Asai, P. Reddy, and J. C. Cuevas, New J. Phys. 16, 015004 (2014).

${ }^{70}$ L. Venkataraman, Y. S. Park, A. C. Whalley, C. Nuckolls, M. S. Hybertsen, and M. L. Steigerwald, Nano Lett. 7, 502 (2007).

71 M. T. González, A. Díaz, E. Leary, R. García, M. A. Herranz, G. Rubio-Bollinger, N. Martín, and N. Agraït, J. Am. Chem. Soc. 135, 5420 (2013).

72 Q. Lu, K. Liu, H. Zhang, Z. Du, X. Wang, and F. Wang, ACS Nano 3, 3861 (2009). 